\title{
Cycloastragenol alleviates airway inflammation in asthmatic mice by inhibiting autophagy
}

\author{
XUEYI ZHU ${ }^{1 *}$, YUXUE CAO ${ }^{1,2^{*}}$, MINGYUE SU ${ }^{3}$, MENGMENG CHEN $^{1}$, CONGCONG LI $^{1}$, \\ LA YI $^{1}$, JINGJING QIN ${ }^{1}$, WUNIQIEMU TULAKE ${ }^{1}$, FANGZHOU TENG ${ }^{1}$, YUANYUAN ZHONG ${ }^{1}$, \\ WEIFENG TANG $^{1}$, SHIYUAN WANG ${ }^{1}$ and JINGCHENG DONG ${ }^{1,2}$ \\ ${ }^{1}$ Department of Integrative Medicine, Huashan Hospital, Fudan University; ${ }^{2}$ Institutes of Integrative \\ Medicine, Fudan University, Shanghai 200040; ${ }^{3}$ Department of Pulmonary Diseases and Oncology, \\ Pu'er Hospital of Traditional Chinese Medicine, Kunming, Yunnan 665000, P.R. China
}

Received May 9, 2021; Accepted August 11, 2021

DOI: $10.3892 / \mathrm{mmr} .2021 .12445$

\begin{abstract}
Cycloastragenol (CAG), a secondary metabolite from the roots of Astragalus zahlbruckneri, has been reported to exert anti-inflammatory effects in heart, skin and liver diseases. However, its role in asthma remains unclear. The present study aimed to investigate the effect of CAG on airway inflammation in an ovalbumin (OVA)-induced mouse asthma model. The current study evaluated the lung function and levels of inflammation and autophagy via measurement of airway hyperresponsiveness (AHR), lung histology examination, inflammatory cytokine measurement and western blotting, amongst other techniques. The results demonstrated that CAG attenuated OVA-induced AHR in vivo. In addition, the total number of leukocytes and eosinophils, as well as the secretion of inflammatory cytokines, including interleukin (IL)-5, IL-13 and immunoglobulin E were diminished in bronchoalveolar lavage fluid of the OVA-induced murine asthma model. Histological analysis revealed that CAG suppressed inflammatory cell infiltration and goblet cell secretion. Notably, based on molecular docking simulation, CAG was demonstrated to bind to the active site of autophagy-related gene 4-microtubule-associated proteins light chain 3 complex, which explains the reduced autophagic flux in asthma caused by CAG. The expression levels of proteins associated with autophagy pathways were inhibited following treatment with CAG. Taken together, the results of the present study suggest that CAG exerts an anti-inflammatory effect in asthma, and its role may be associated with the inhibition of autophagy in lung cells.
\end{abstract}

Correspondence to: Dr Jingcheng Dong, Department of Integrative Medicine, Huashan Hospital, Fudan University, 12 Middle Urumqi Road, Shanghai 200040, P.R. China

E-mail: jcdong2004@126.com

*Contributed equally

Key words: cycloastragenol, asthma, airway inflammation, autophagy, light chain 3, p62

\section{Introduction}

Asthma is a common chronic inflammatory disease in the respiratory system that is characterized by wheeze, shortness of breath, chest tightness, cough and obvious expiratory airflow limitation (1). Previous statistics have indicated that $>334$ million individuals suffer from asthma, at the risk of high mortality and global economic burden (2). Airway hyperresponsiveness (AHR), airway inflammation, inflammatory cytokine infiltration and mucus hypersecretion are commonly associated with asthma (3). T helper (Th) 2 cell-mediated type 2 inflammation and eosinophil abundance are associated with the progression and exacerbation of asthma $(4,5)$. Currently, inhaled corticosteroids and bronchodilators are used as the main treatment for asthma (6). However, some asthmatic patients who overuse these may suffer from various side effects, including osteoporosis, infection and drug dependence (7). Therefore, it is important to identify other effective and safe therapeutics for asthmatic patients.

Autophagy, like self-eating, is involved in the innate and adaptive immune responses of asthma (8). In the presence of allergens, damaged proteins and organelles decompose themselves, which are captured by autophagosomes and degraded by lysosomes to achieve the immune balance in asthma (9). It has been proposed that in the initiation of autophagy, Beclin 1, a major activator of autophagy (10), participates in the recruitment of autophagy proteins to form autophagosomes (11). Microtubule-associated proteins light chain (LC) 3 is hydrolyzed to LC3I by autophagy-related gene (ATG) 4 , which is subsequently hydrolyzed to LC3II during autophagosome formation (12). Furthermore, sequestosome 1 (SQSTM1/p62, p62) can reflect the level of autophagy, and a reduced level of p62 is generally considered as a marker of activated autophagy, as the enhancement of autophagy leads to the degradation of the stress-inducible cellular adapter protein p62 (13). The stable state of autophagy can regulate both energy homeostasis and the quality of proteins and organelles in airway inflammation (14). Otherwise, overactivation of autophagy causes the deterioration of inflammation (15). Previous studies reported that autophagy is activated in asthma and acts as a double-edged sword, whereby either enhancement 
or decrease of autophagy can ease the airway inflammation of asthma $(16,17)$. Liu et al (18) proved that inhibition of autophagy alleviates airway inflammation and AHR in severe asthmatic mice. Furthermore, McAlinden et al (19) demonstrated that in the airway remodeling of asthma, the autophagy inhibitor represses airway smooth muscle proliferation and profibrotic signaling. However, the specific mechanism by which autophagy mediates the airway inflammation of asthma remains unclear.

Traditional Chinese medicine is an effective method to treat asthma (20). A series of studies $(21,22)$ have reported that Astragalus membranaceus (huangqi) has anti-asthma effects, whereby it reduces inflammatory cytokines and improves efficacy by modulating immune balance. Aastragaloside IV, as the main active component of Astragalus membranaceus also exerts strong anti-allergic effects (23), whereby it protects mice with allergic rhinitis from inflammation (24), enhances Th1-associated anti-inflammatory cytokines and diminishes Th2-associated pro-inflammatory cytokines (25). Cycloastragenol (CAG), as the main metabolite of Astragaloside IV in vivo, is a potent small molecule telomerase activator (26). CAG has been reported to exert anti-inflammatory effects in cardiovascular, hepatic, skin and aging diseases $(27,28)$. Notably, CAG is also considered a modulator of autophagy, associated with the balance between pro-inflammation and anti-inflammation. However, whether CAG regulates airway inflammatory conditions remains unclear as the anti-asthmatic effects of CAG have not yet been investigated. Thus, the present study used ovalbumin (OVA)-induced asthmatic mice to investigate the anti-inflammatory effects of CAG in asthma and determine its potential molecular mechanisms.

\section{Materials and methods}

Animals. A total of $20 \mathrm{BALB} / \mathrm{c}$ female mice [6-weeks-old (29); body weight, $18 \pm 2 \mathrm{~g}$ ] were purchased from Jiesijie Laboratory Animal Co., Ltd. [license no. SYXK(Hu)2020-0032; http://www.jsj-lab.com/]. All mice were maintained under specific pathogen-free conditions with a 12-h light/dark cycle and a free access to food and water at a controlled temperature of $22 \pm 2^{\circ} \mathrm{C}$ with $55 \%$ relative humidity. All animal experiments were ethically reviewed and approved by the Animal Care and Use Committee of the Fudan University (authorization no. 201 8-10-HSYY-DJC-01; Shanghai, China).

OVA-induced asthmatic mice and treatment. Age- and sex-matched BALB/c mice were randomly divided into five groups (4 mice/group), including the normal control (NC), OVA-induced asthma model (Asthma), low CAG dose (Asthma/31.25 mg/kg CAG), middle CAG dose (Asthma/62.5 mg/kg CAG) and high CAG dose (Asthma/125 mg/kg CAG) groups. The OVA and three doses of CAG groups were immunized on days 0,7 and 14 intraperitoneally by OVA (100 ug/mouse, grade V, Sigma-Aldrich; Merck KGaA) mixed with $10 \mathrm{mg}$ aluminum hydroxide (Thermo Fisher Scientific, Inc.), which was dissolved in $0.2 \mathrm{ml}$ sterile saline. Furthermore, mice were intranasally challenged with $50 \mu \mathrm{g}$ OVA (dissolved in 50 $\mu$ 1 PBS) on days 21-25 (30-32). The NC group was immunized with saline and challenged by
PBS instead. CAG (Winherb Medical Technology Co., Ltd.; https://www.sh-winherb.com/Index.aspx) was respectively administrated to the three doses of CAG groups intragastrically (dissolved in $0.2 \mathrm{ml} 0.5 \%$ sodium carboxymethyl cellulose/mouse) on days 21-25. The mice were anesthetized with $2 \%$ phenobarbital sodium $(50 \mathrm{mg} / \mathrm{kg})$ intraperitoneally and sacrificed after $24 \mathrm{~h}$.

Measurement of AHR. The mice were tracheostomized, intubated and placed in a single-chamber, whole-body plethysmograph connected to the ventilator (DSI Buxco Electronics; https:/www.datasci.com/products/buxco-respiratory-products/ finepointe-resistance-and-compliance). To evaluate airway responsiveness, changes in total lung resistance $\left(R_{L}\right)$ and dynamic lung compliance (Cdyn) were measured in response to aerosolized methacholine (Mch, Sigma-Aldrich; Merck KGaA) at increasing doses of $0,6.25,12.5,50$ and $100 \mathrm{mg} / \mathrm{ml}$. The mice were subsequently euthanatized with $2 \%$ phenobarbital sodium $(150 \mathrm{mg} / \mathrm{kg})$ intraperitoneally.

Collection of bronchoalveolar lavage fluid (BALF) and leukocyte classification and counts. Following measurement of AHR, lungs were lavaged using the tracheal cannula (https://www.biomart.cn/infosupply/76901595.htm) with $300 \mu 1$ aliquots of ice-cold PBS twice, and centrifuged at $500 \mathrm{x} \mathrm{g}$ for $10 \mathrm{~min}$ at $4^{\circ} \mathrm{C}$. The supernatants were stored at $-80^{\circ} \mathrm{C}$ until further analyses of cytokines. Total cells were resuspended in $50 \mu \mathrm{l}$ PBS and counted using the Mindray BC-5000Vet automated hematology analyzer (Mindray; https://www.mindray.com/cn/product/BC-5000.html).

Lung histology. At room temperature, lung sections (4\% phosphate-buffered and formalin-fixed for $24 \mathrm{~h}$ ) of the middle lobe of the left lung $(4-\mu \mathrm{m}$ thick) were embedded in paraffin, stained with hematoxylin and eosin (H\&E; cat. no. G1003; Wuhan Servicebio Technology Co., Ltd.) for total $10 \mathrm{~min}$ or periodic acid-schiff (PAS; cat. no. G1008; Wuhan Servicebio Technology Co., Ltd.) for total $40 \mathrm{~min}$ and dehydrated with $100 \%$ ethanol (cat. no. 100092683; Sinopharm Chemical Reagent Co., Ltd.) for 5 min three times and xylene (cat. no. 10023418, SCRC) for 5 min twice, according to the manufacturer's instructions. Inflammation score of $\mathrm{H} \& \mathrm{E}$ staining (33) and the percentage of $\mathrm{PAS}^{+}$bronchial cells (34) were determined as previously described.

ELISA. The levels of interleukin (IL)-5, IL-13 and immunoglobulin $\mathrm{E}(\mathrm{IgE})$ in the BALF were determined using ELISA kits (IL-5, Mouse, cat. no. 70-EK205-48; IL-13, Mouse, cat. no. 70-EK213/2-48; IgE, Mouse, cat. no. 70-EK275-48; MultiSciences), according to the manufacturer's instructions.

Molecular docking simulation. The 3D structure of CAG was obtained from PubChem Compound (https://www.ncbi.nlm. nih.gov/pccompound, PubChem CID: 13943286) (35). The X-ray crystal structure of the ATG4-LC3 complex [Protein Data Bank (PDB) ID: 2Z0D] was acquired from RCSB PDB (https://www.rcsb.org) (36). Subsequently, both of them were converted into pdbqt formats via AutoDockTools 1.5.6 (37) and were optimized by removing water molecules and adding 
polar hydrogen atoms. The potential binding sites of the ATG4-LC3 complex within CAG were determined using the molecular docking study employing the docking program AutoDock Vina (38). The coordinates of the target active pocket were center_x $=-5.906$, center_y $=-15.694$ and center_ $\mathrm{z}=27.844$. Size $\_\mathrm{x}=40$, size $\_\mathrm{y}=40$ and size $\_\mathrm{z}=40$ were applied. The docking process was also calculated using AutoDock Vina (all default values). The highly scored docking result was visualized using PyMoL 2.3.0 (39) and Discovery Studio 2017 R2 Client (40).

Western blotting. To extract protein, lung tissues were minced and homogenized in ice-cold RIPA lysis buffer containing phosphatase inhibitors and a protease inhibitor (Beyotime Institute of Biotechnology) and centrifuged at $14,000 \mathrm{x} \mathrm{g}$ for $10 \mathrm{~min}$ at $4^{\circ} \mathrm{C}$. Protein concentrations were quantified using the Pierce BCA Protein Assay kit (Thermo Fisher Scientific, Inc.). Protein $(30 \mu \mathrm{g})$ was loaded into each well and separated via $12 \%$ SDS-PAGE. The separated proteins were transferred onto $0.45 \mu \mathrm{m}$ PVDF membranes and blocked with $5 \%$ milk for $1 \mathrm{~h}$ at room temperature. The membranes were incubated with the following primary antibodies; Anti-rabbit LC3B (1:1,000; cat. no. 3868S; Cell Signaling Technology, Inc.), anti-rabbit SQSTM1/p62 (1:1,000; cat. no. 5114T; Cell Signaling Technology, Inc.) and anti-rabbit Beclin 1 (1:1,000; cat. no. 11306-1-AP; ProteinTech Group, Inc.) overnight at $4{ }^{\circ} \mathrm{C}$. Following the primary incubation, membranes were incubated with HRP-conjugated secondary antibodies (1:10,000; cat. no. SA00001-2; ProteinTech Group, Inc.) for $1.5 \mathrm{~h}$ at room temperature. Protein bands were visualized using ImageQuant LAS-4000 mini (Cytiva) and analyzed using ImageJ 1.53 software (National Institutes of Health).

Immunohistochemistry. Paraffin-embedded sections of lungs (4\% phosphate-buffered and formalin-fixed for $24 \mathrm{~h}$ at room temperature; $4-\mu \mathrm{m}$ thick) were dewaxed in xylene, rehydrated in ethanol and blocked with 3\% BSA (cat. no. G5001; Wuhan Servicebio Technology Co., Ltd.) for $30 \mathrm{~min}$ at room temperature. Following antigen retrieval, sections were incubated with anti-LC3B antibody (1:300; cat. no. 14600-1-AP; ProteinTech Group, Inc.), anti-SQSTM1/p62 antibody (1:400; cat. no. 88588S; Cell Signaling Technology, Inc.) and anti-Beclin 1 antibody (1:400; cat. no. 11306-1-AP; ProteinTech Group, Inc.) for $12 \mathrm{~h}$ at $4^{\circ} \mathrm{C}$. Then, sections were incubated with HRP-conjugated secondary antibodies (1:200; cat. no. GB23303; Wuhan Servicebio Technology Co., Ltd.) for $50 \mathrm{~min}$ at $24^{\circ} \mathrm{C}$. Cells were counted in five randomly selected fields using an optical microscope (magnification, $\mathrm{x} 400$ ) and analysis was performed using ImageJ 1.53 software (National Institutes of Health), as previously described (41).

Statistical analysis. Statistical analysis was performed using GraphPad Prism 8.0 software (GraphPad Software, Inc.). Each experiment was repeated $\geq 3$ times. Data are presented as the mean \pm SEM. One-way ANOVA followed by Tukey's post hoc test was used for intragroup and intergroup comparisons $(42,43)$. Kruskal-Wallis test followed by Dunn's post hoc test was used for infla0mmation score. $\mathrm{P}<0.05$ was considered to indicate a statistically significant difference.

\section{Results}

CAG attenuates AHR in the OVA-induced murine asthma model. Mice were sensitized, intranasally challenged and administrated treatment according to the protocol presented in Fig. 1A. At $24 \mathrm{~h}$ after the final OVA challenge, lung function was evaluated through direct measurements of $\mathrm{R}_{\mathrm{L}}$ and Cdyn. The results demonstrated that compared with the $\mathrm{NC}$ group, dose-dependent increases of $\mathrm{R}_{\mathrm{L}}$ at doses $6.25(\mathrm{P}<0.05), 12.5$ $(\mathrm{P}<0.01), 50(\mathrm{P}<0.01)$ and $100 \mathrm{mg} / \mathrm{ml} \mathrm{Mch}(\mathrm{P}<0.001)$ were observed in the Asthma group (Fig. 1B), as well as significant dose-dependent declines of Cdyn at doses of $6.25(\mathrm{P}<0.05)$, $12.5(\mathrm{P}<0.01), 50(\mathrm{P}<0.05)$ and $100 \mathrm{mg} / \mathrm{ml}$ Mch $(\mathrm{P}<0.001)$ (Fig. 1C). Alternatively, compared with the Asthma group, notable reductions of $\mathrm{R}_{\mathrm{L}}(\mathrm{P}<0.05$; Fig. $1 \mathrm{~B})$ and enhancements of Cdyn $(\mathrm{P}<0.05$; Fig. 1C) were observed in both the Asthma $/ 62.5 \mathrm{mg} / \mathrm{kg}$ CAG and Asthma $/ 125 \mathrm{mg} / \mathrm{kg}$ CAG groups at doses of 12.5 and $50 \mathrm{mg} / \mathrm{ml} \mathrm{Mch}$. Notably, at $100 \mathrm{mg} / \mathrm{ml}$ Mch, significant decreases of $R_{L}$ (Fig. 1D) and elevations of Cdyn (Fig. 1E) were observed in the Asthma $/ 62.5 \mathrm{mg} / \mathrm{kg}$ CAG $(\mathrm{P}<0.05)$ and Asthma/125 mg/kg CAG $(\mathrm{P}<0.01)$ groups. Although the Cdyn of mice in the Asthma $/ 31.25 \mathrm{mg} / \mathrm{kg}$ CAG group increased at $100 \mathrm{mg} / \mathrm{ml} \mathrm{Mch}(\mathrm{P}<0.05)$, the low dose of CAG had no significant effect on the decrease of $R_{L}$. Taken together, these results suggest that 62.5 and $125 \mathrm{mg} / \mathrm{kg}$ CAG have the ability to attenuate AHR and improve dynamic lung compliance, particularly $125 \mathrm{mg} / \mathrm{kg}$ CAG.

CAG alleviates immune cell abundance and eosinophil recruitment. To investigate whether CAG effects immune cells in asthma, BALF was collected and inflammatory cell classification and counts were determined. The results demonstrated that compared with the NC group, the Asthma group displayed significantly higher numbers of total leucocytes $(\mathrm{P}<0.01)$, neutrophils $(\mathrm{P}<0.05)$, lymphocytes $(\mathrm{P}<0.05)$, monocytes $(\mathrm{P}<0.05)$ and eosinophils $(\mathrm{P}<0.01)$. Notably, $125 \mathrm{mg} / \mathrm{kg}$ CAG suppressed the levels of these cells $(\mathrm{P}<0.05)$, particularly eosinophils $(\mathrm{P}<0.01)$. Notably, $62.5 \mathrm{mg} / \mathrm{kg}$ CAG significantly inhibited the levels of total leucocytes and eosinophils $(\mathrm{P}<0.05)$ compared with the Asthma group (Fig. 2A).

CAG decreases inflammatory cell infiltration and mucus hypersecretion. To assess the inflammation of bronchus in lung tissues, histological changes were detected via H\&E (Fig. 2B) and PAS (Fig. 2C) staining. After OVA induction, there was excessive mucus secretion in the Asthma group, while this elevated mucus secretion was reversed in the $125 \mathrm{mg} / \mathrm{kg}$ CAG group (Fig. 2C). According to the H\&E staining results, inflammatory cells were significantly infiltrated in the Asthma group compared with the NC group $(\mathrm{P}<0.05)$. Notably, only $125 \mathrm{mg} / \mathrm{kg}$ CAG significantly suppressed inflammation compared with the Asthma group ( $\mathrm{P}<0.05$; Fig. 2D). The PAS staining results demonstrated that the $\mathrm{PAS}^{+}$bronchial cell count significantly increased in the Asthma group following OVA induction $(\mathrm{P}<0.001$; Fig. $2 \mathrm{E})$, which suggests that mucus secretion of the Asthma group is extremely excessive. As expected, $125 \mathrm{mg} / \mathrm{kg}$ CAG significantly relieved mucus secretion $(\mathrm{P}<0.05)$. Although the low dose of $31.25 \mathrm{mg} / \mathrm{kg}$ CAG had modest relief in both lung function and airway inflammation, the tendency was not significant. Thus, according to the 

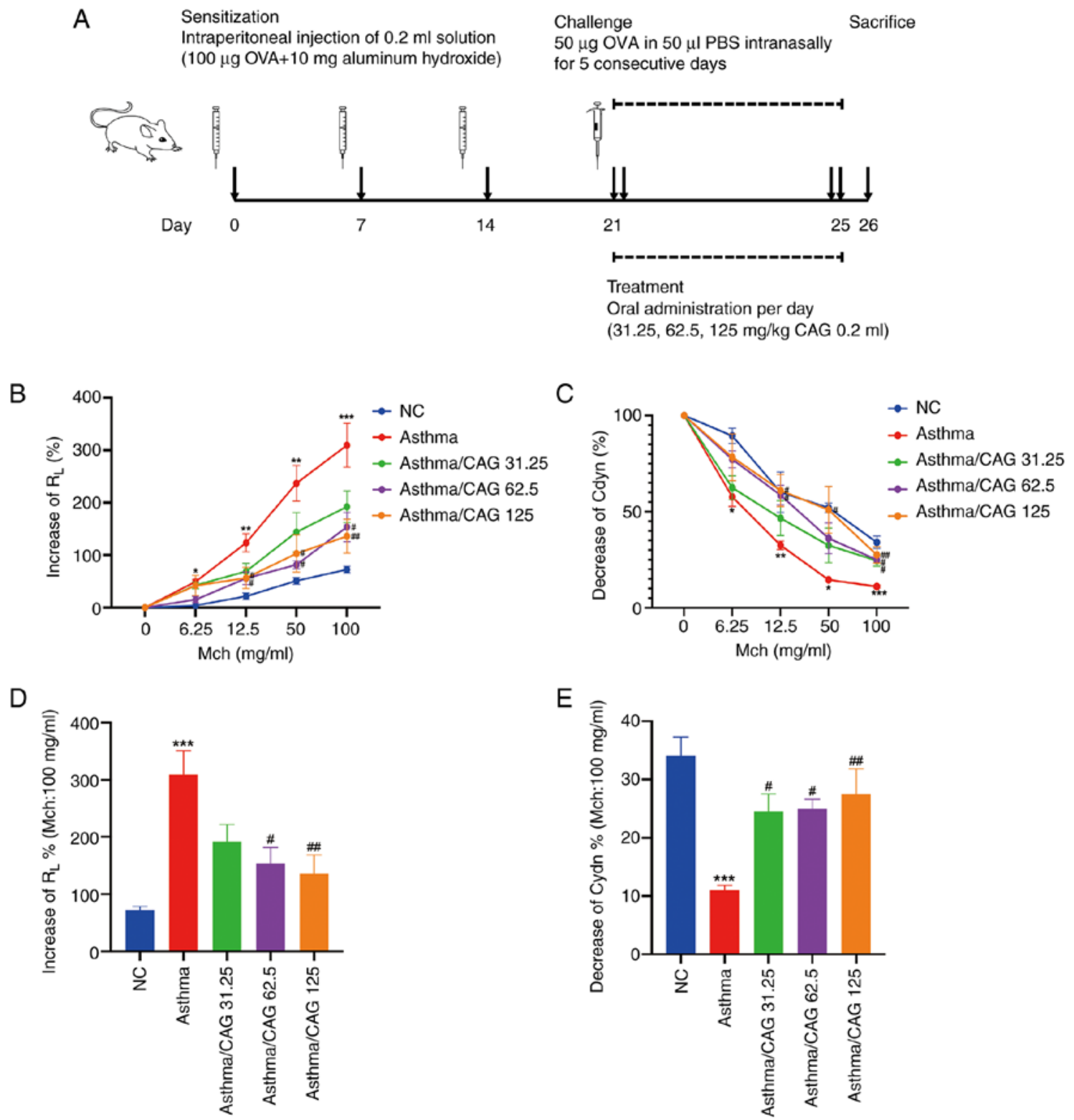

Figure 1. Effects of CAG on airway hyperresponsiveness to Mch in OVA-induced asthmatic mice ( $\mathrm{n}=4$ mice/group). (A) Asthma induction and administration protocols in murine. (B) $\mathrm{R}_{\mathrm{L}} \%$ and (C) Cdyn $\%$ to Mch. (D) $\mathrm{R}_{\mathrm{L}} \%$ and (E) Cdyn $\%$ to $100 \mathrm{mg} / \mathrm{ml} \mathrm{Mch}$. Data are presented as the mean $\pm \mathrm{SEM}$. ${ }^{*} \mathrm{P}<0.05$, ${ }^{* *} \mathrm{P}<0.01$, ${ }_{* * * *} \mathrm{P}<0.001$ vs. the $\mathrm{NC}$ group; ${ }^{*} \mathrm{P}<0.05,{ }^{\# \#} \mathrm{P}<0.01$ vs. the Asthma group. $\mathrm{CAG}$, cycloastragenol; Mch, methacholine; OVA, ovalbumin; $\mathrm{R}_{\mathrm{L}}$, total lung resistance; Cdyn, dynamic lung compliance; NC, negative control.

results of the measurement of $\mathrm{R}_{\mathrm{L}}$, the counts of total cells and eosinophils and H\&E staining, 62.5 and $125 \mathrm{mg} / \mathrm{kg} \mathrm{CAG}$ were selected for subsequent experimentation.

$C A G$ alleviates inflammatory cytokines and IgE in BALF. The effects of 62.5 and $125 \mathrm{mg} / \mathrm{kg}$ CAG on the levels of Th2 cytokines and IgE, which are common in allergic asthma (44), were investigated. The results demonstrated that the levels of IL-5 $(\mathrm{P}<0.05$; Fig. 3A), IL-13 ( $\mathrm{P}<0.01$; Fig. 3B) and $\operatorname{IgE}(\mathrm{P}<0.01$; Fig. 3C) were significantly higher in the Asthma group compared with the NC group. Notably, $125 \mathrm{mg} / \mathrm{kg}$ CAG significantly decreased the levels of IL-5 $(\mathrm{P}<0.05)$, IL-13 $(\mathrm{P}<0.05)$ and $\mathrm{IgE}(\mathrm{P}<0.01)$. However, $62.5 \mathrm{mg} / \mathrm{kg} \mathrm{CAG}$ significantly decreased the level of IL-5 $(\mathrm{P}<0.05)$. Collectively, these results suggest that $125 \mathrm{mg} / \mathrm{kg}$ CAG regulates $\mathrm{Th} 2$-associated inflammation.
Molecular docking study. After confirming the anti-inflammatory function of CAG (Fig. 4A) in asthma, the present study investigated the specific mechanism and performed molecular docking between the ATG4-LC3B complex and CAG to determine whether CAG can modulate autophagy-related proteins, and the potential interaction between them. The highest binding energy of CAG towards the ATG4-LC3B complex was $-8.0 \mathrm{kcal} / \mathrm{mol}$, and the docking analysis predicted that CAG made hydrogen-bonding interactions with LEU232 and GLN43 at the active site (Fig. 4B). Furthermore, CAG probably formed a pi-alkyl with LYS42.

CAG inhibits autophagy-related proteins in lung tissues. To further verify the regulation of CAG on the expression of autophagy-related proteins, the present study examined the major autophagy-related factors, LC3B, p62 and Beclin 1. 

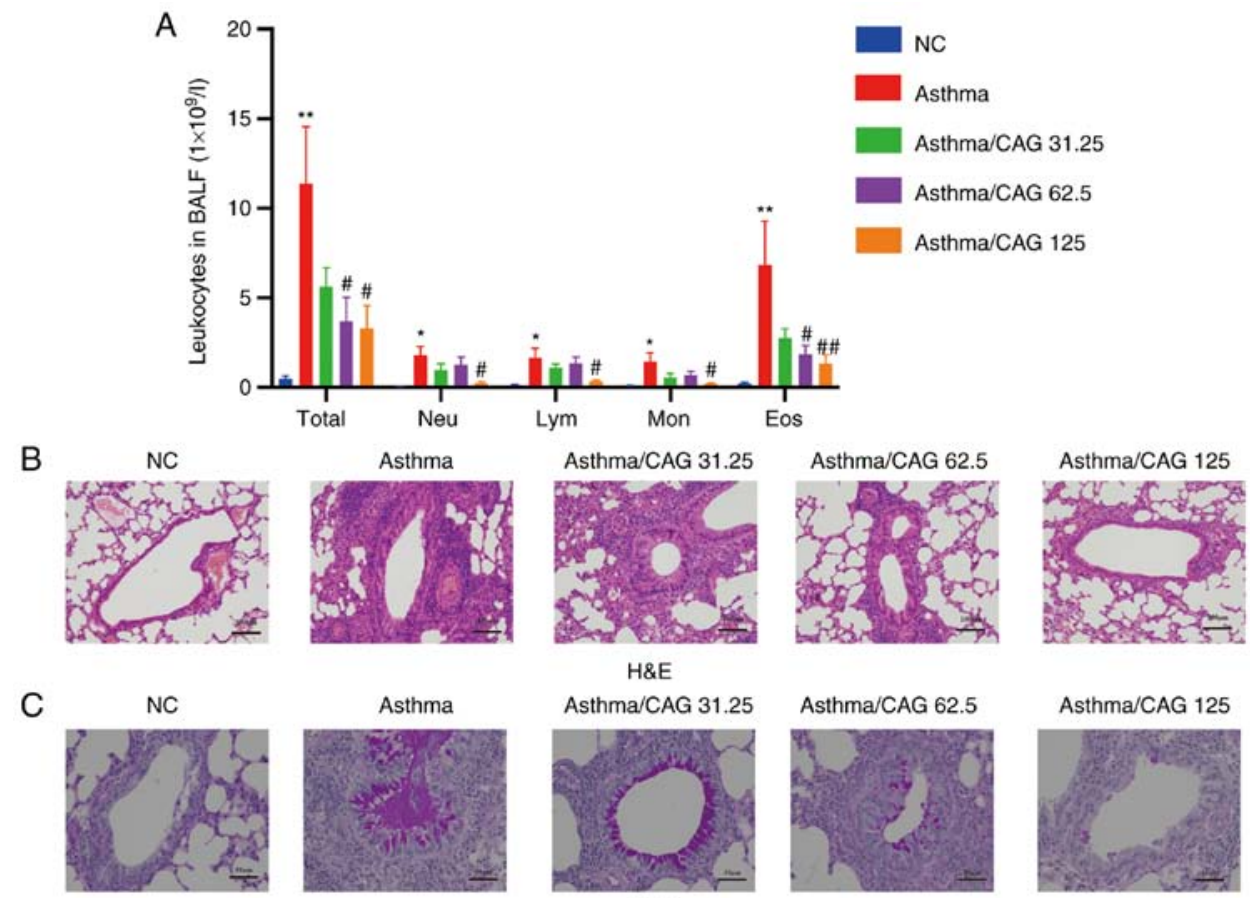

Asthma/CAG 31.25
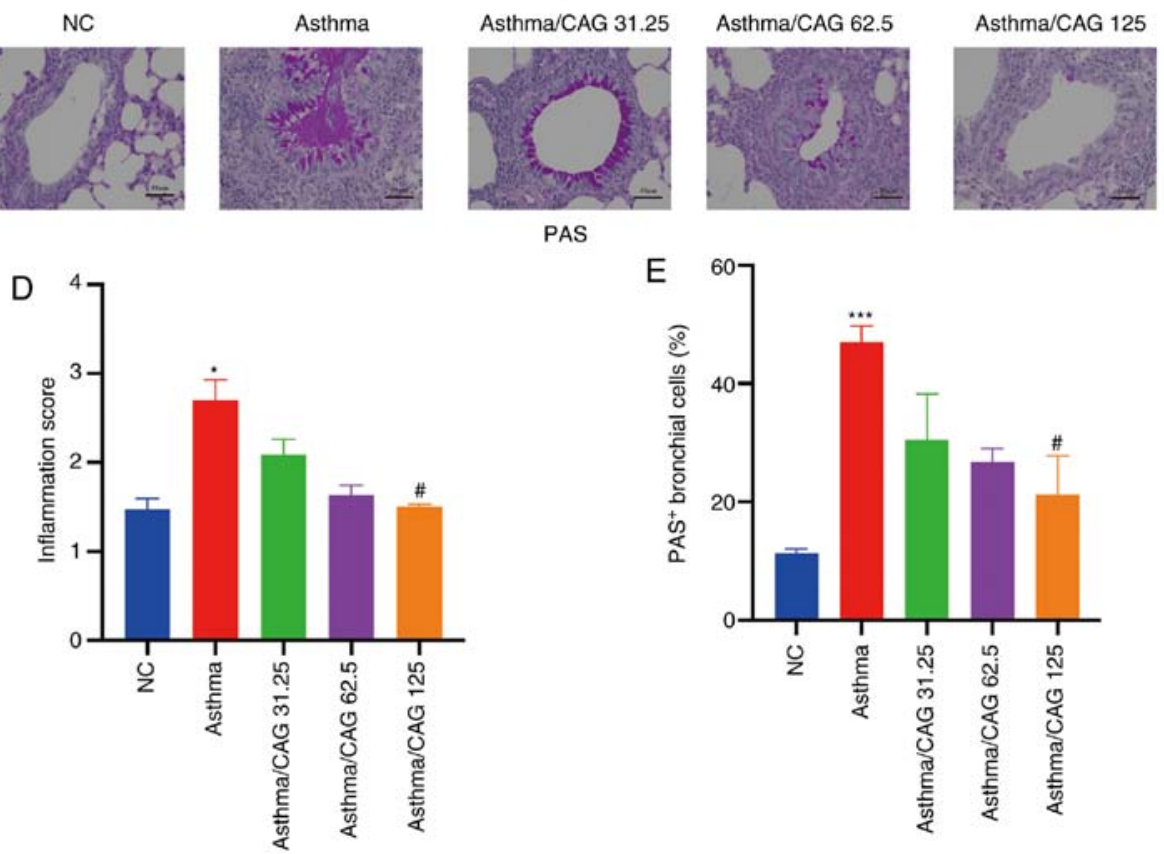

Figure 2. Effects of CAG on inflammatory cells, airway inflammation and mucus hypersecretion in ovalbumin-induced asthmatic mice (n=4 mice/group). (A) Number of Total, Neu, Lym, Mon and Eos in BALF. (B) Histological examination of H\&E staining (magnification, x100; scale bar, $100 \mu \mathrm{m}$ ). (C) Histological examination of PAS staining (magnification, $\mathrm{x} 200$; scale bar, $50 \mu \mathrm{m}$ ). (D) Inflammation score acquired with H\&E staining. (E) The percentage of PAS ${ }^{+}$bronchial cells. Data are presented as the mean \pm SEM. ${ }^{*} \mathrm{P}<0.05,{ }^{* *} \mathrm{P}<0.01,{ }^{* * * *} \mathrm{P}<0.001$ vs. the $\mathrm{NC}$ group; ${ }^{*} \mathrm{P}<0.05,{ }^{\# \#} \mathrm{P}<0.01$ vs. the Asthma group. CAG, cycloastragenol; Total, total leucocytes; Neu, neutrophils; Lym, lymphocytes; Mon, monocytes; Eos, eosinophils; BALF, bronchoalveolar lavage fluid; H\&E, hematoxylin and eosin; PAS, periodic acid-schiff; NC, negative control.
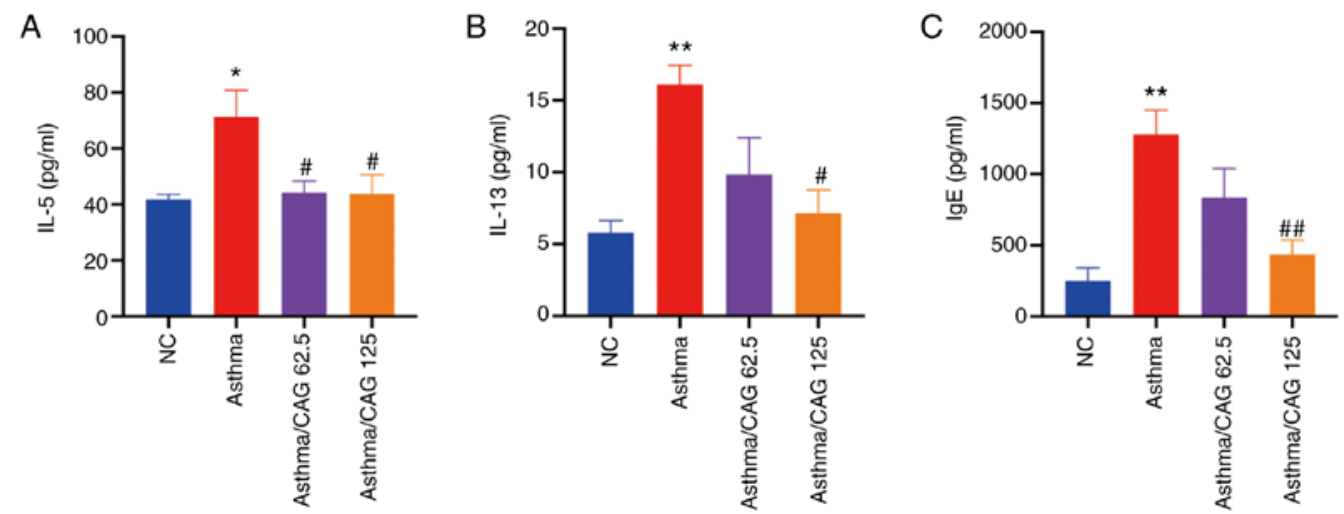

Figure 3. Effects of CAG on inflammatory cytokines and IgE in bronchoalveolar lavage fluid ( $\mathrm{n}=4$ mice/group). The expression levels of (A) IL-5, (B) IL-13 and (C) IgE. Data are presented as the mean \pm SEM. ${ }^{~} \mathrm{P}<0.05,{ }^{* *} \mathrm{P}<0.01$ vs. the $\mathrm{NC}$ group; ${ }^{\#} \mathrm{P}<0.05,{ }^{\# \#} \mathrm{P}<0.01$ vs. the Asthma group. CAG, cycloastragenol; IgE, immunoglobulin E; IL, interleukin; NC, negative control. 
A

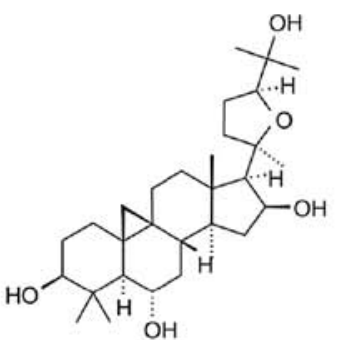

Cycloastragenol

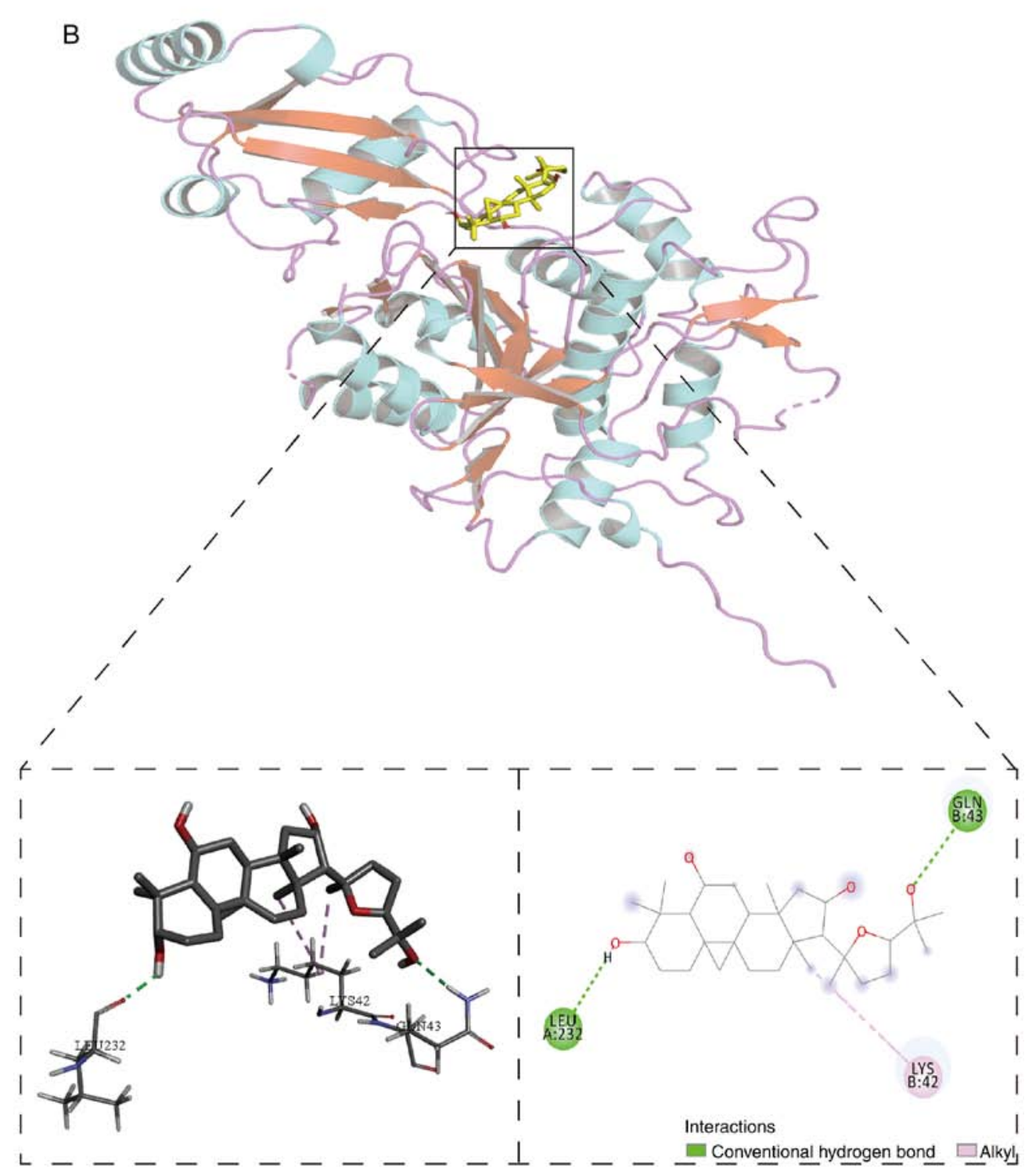

Figure 4. Molecular docking simulation of CAG. (A) Molecular structure of CAG. (B) The potential molecular interaction of the potent phytochemical, CAG, with target ATG4-LC3 complex (PDB ID: 2Z0D), from the docking study. PyMoL was used to visualize both 3D and 2D formats. CAG, cycloastragenol; ATG4-LC3, autophagy-related gene 4 light chain 3; D, dimensional.

According to the results of western blotting, it was found that the expression levels of LC3B and Beclin 1 were enhanced, while the expression of p62 was diminished in the Asthma group. Moreover, $125 \mathrm{mg} / \mathrm{kg}$ CAG restored these expressions (Fig. 5A). The results demonstrated that LC3B protein expression was significantly higher in the Asthma group compared with the NC group ( $\mathrm{P}<0.01$; Fig. 5B). Furthermore, p62 protein expression was significantly lower in the Asthma group compared with the NC group $(\mathrm{P}<0.01$; Fig. $5 \mathrm{C})$.
Notably, LC3B protein expression was relieved following treatment with $62.5 \mathrm{mg} / \mathrm{kg}$ CAG $(\mathrm{P}<0.05)$ and $125 \mathrm{mg} / \mathrm{kg}$ CAG, particularly in the higher dose $(\mathrm{P}<0.01)$. In addition, $62.5 \mathrm{mg} / \mathrm{kg}$ CAG $(\mathrm{P}<0.05)$ and $125 \mathrm{mg} / \mathrm{kg}$ CAG $(\mathrm{P}<0.01)$ restored the p62 expression, which suggests that high doses of CAG can significantly inhibit autophagy in the asthma model. Immunohistochemistry analysis (Fig. 5D-F) revealed a notable increase in the expression levels of LC3B $(\mathrm{P}<0.0001$; Fig. $5 \mathrm{G})$ and Beclin $1(\mathrm{P}<0.05)$ (Fig. 5I) with OVA challenge, while 

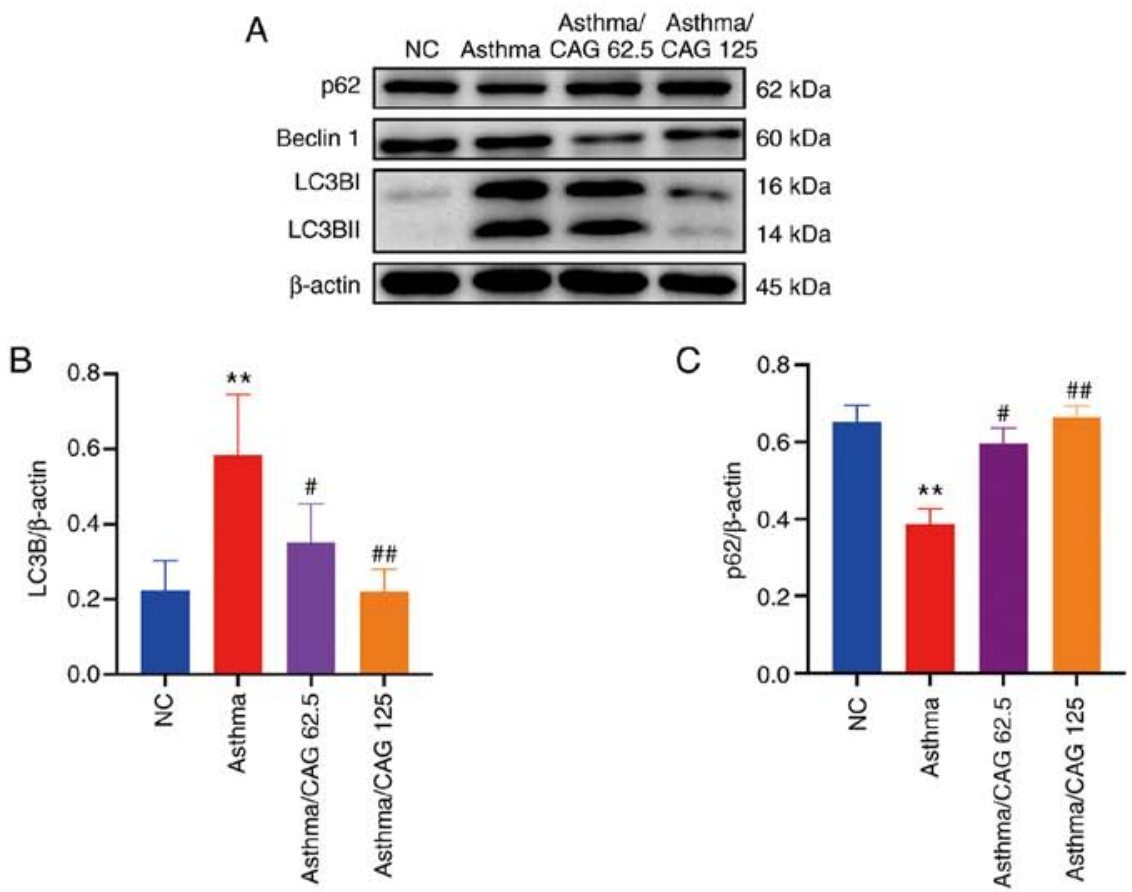

D

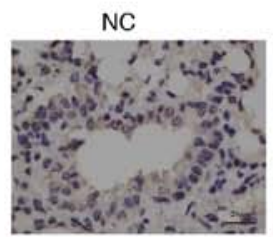

Asthma
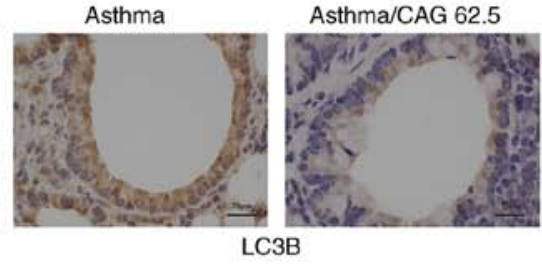

Asthma/CAG 125

E
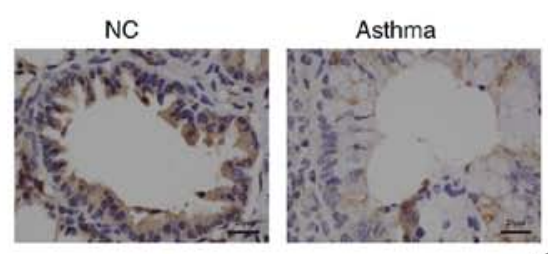

Asthma/CAG 62.5

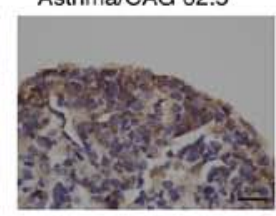

Asthma/CAG 125

$\mathrm{F}$
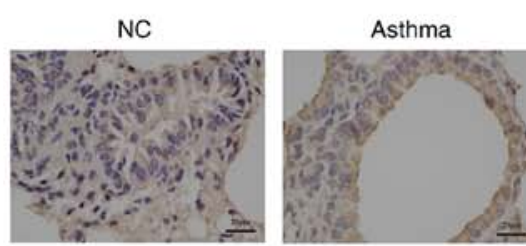

Asthma/CAG 62.5

Asthma/CAG 125
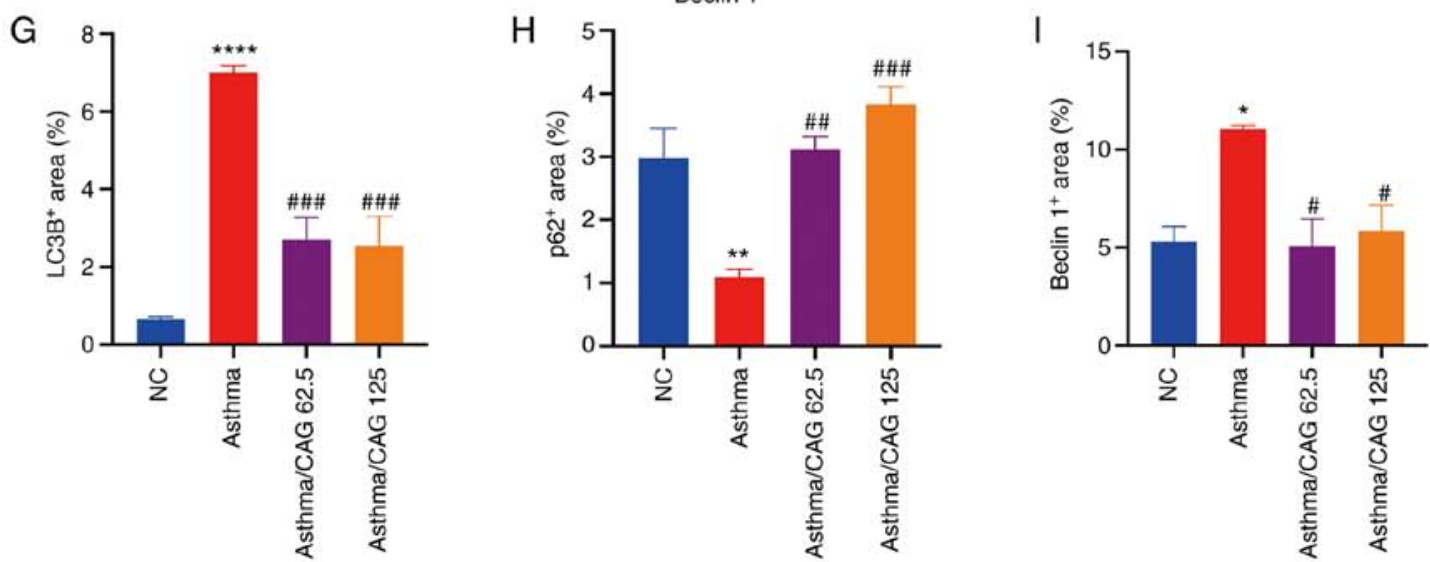

Figure 5. Effects of CAG on the expression levels of LC3B, p62 and Beclin 1 in ovalbumin-induced asthmatic mice (n=4 mice/group). (A) Protein expression levels of LC3B, p62 and Beclin 1 in the assessed groups. Relative density quantifications of (B) LC3B and (C) p62. Data are presented as a ratio of LC3B and p62 relative to $\beta$-actin. Immunohistochemistry analysis for positive expression levels of (D) LC3B, (E) p62 and (F) Beclin 1 (magnification, x400; scale bar, $100 \mu \mathrm{m}$ ). Positive areas of protein expression levels of (G) LC3B, (H) p62 and (I) Beclin 1. Data are presented as the mean \pm SEM. "P<0.05, **P $<0.01$,

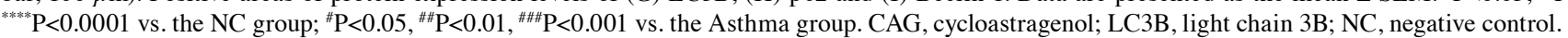




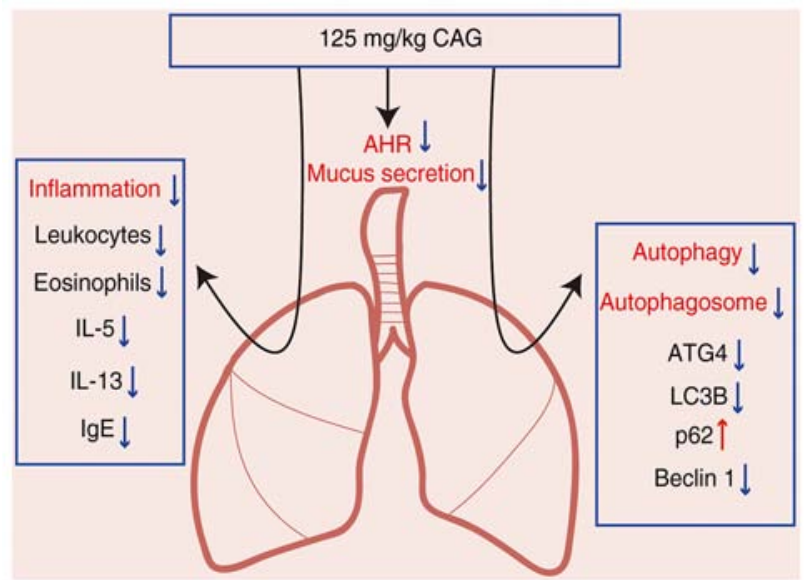

Figure 6. Schematic diagram of the probable mode of effects of CAG on asthma. CAG suppresses asthma phenotypes in OVA-induced mice. The molecular mechanisms underlying the inhibitory effects of CAG on OVA-induced asthma are suggested to involve repression on AHR, airway inflammation and mucus hypersecretion, as well as the suppression of autophagy. CAG, cycloastragenol; OVA, ovalbumin; AHR, airway hyperresponsiveness; IL, interleukin; IgE, immunoglobulin E; ATG4, autophagy-related gene 4; LC3B, light chain 3B.

p62 expression significantly decreased in the Asthma group $(\mathrm{P}<0.01$; Fig. 5H). Taken together, these results confirm that 62.5 and $125 \mathrm{mg} / \mathrm{kg}$ CAG decrease LC3B expression $(\mathrm{P}<0.001)$ and Beclin 1 expression $(\mathrm{P}<0.05)$. In addition, $125 \mathrm{mg} / \mathrm{kg} \mathrm{CAG}$ significantly increased p62 expression $(\mathrm{P}<0.001)$, which were consistent with the western blot results. Overall, $125 \mathrm{mg} / \mathrm{kg}$ CAG had the potential to alleviate the levels of inflammation to attenuate AHR and mucus secretion in asthma pathogenesis, probably via the inhibition of the levels of autophagy (Fig. 6).

\section{Discussion}

Asthma is characterized by airway inflammation, AHR and airway remodeling (45). Although corticosteroids are used to treat airway inflammation of asthma, they still have multiple adverse reactions, such as infections, diabetes and osteoporosis (46). Thus, other safe and effective therapies are required to relieve inflammation of asthma that contribute to improved quality of life and reduce social burden.

CAG, an active sapogenin of Astragaloside IV, has been proposed to function on multiple pharmacological effects and has been gradually developed as a modern dietary ingredient (47). Recent studies (48-52) have demonstrated that CAG exerts protective effects in inflammation and oxidation. However, whether CAG can prevent the progress of asthma in murine remains unknown. Thus, the present study investigated the course of airway inflammation in asthma and established an OVA-induced acute asthmatic murine model to assess the anti-asthmatic effect of CAG in vivo. Notably, all mice survived and had no loss of body weight. Furthermore, AHR, immune cell infiltration and the metaplasia and hypersecretion of goblet cells were restored via CAG, potentially through inhibition of autophagy.

$R_{L}$ and Cdyn reflect the state of lung ventilation, whereby high $\mathrm{R}_{\mathrm{L}}$ is associated with airflow obstruction of main bronchus and low Cdyn is associated with narrowing of peripheral bronchus (53). The results of the present study confirmed that both 62.5 and $125 \mathrm{mg} / \mathrm{kg}$ CAG triggered the notable decrease of $\mathrm{R}_{\mathrm{L}}$ and elevation of Cdyn, which ameliorated the aggravation of lung function in asthma.

Immune cell counts, as well as H\&E and PAS staining, are the main indicators of airway inflammation and mucus production (54). Overactivation of immune cells, particularly eosinophil recruitment and infiltration promote the progress of asthma (55). In addition, hyperplastic goblet cells produce excessive mucus plugs, exudation and cell debris to cause further airway occlusion (56). It has been demonstrated that high doses of CAG can suppress immune cells to prevent the development of asthma (50); however, $31.25 \mathrm{mg} / \mathrm{kg}$ CAG had little efficacy in both lung function and immune cell counts. Similar results were observed following H\&E and PAS staining.

Then we observed the effects of $62.5 \mathrm{mg} / \mathrm{kg} \mathrm{CAG}$ and $125 \mathrm{mg} / \mathrm{kg}$ CAG on Th2-associated cytokines (IL-5 and IL-13). IL-5 is dominant in Th2-mediated eosinophilic asthma and can reflect the vitality of eosinophils as well as AHR while IL-13 promotes B cells to produce IgE, mucus secretion and exacerbates AHR (57). It was found that they were both repressed by CAG. So, we further measured IgE, a central player in the allergy response, and proved that the enhancement of IgE in asthma was also controlled by CAG (58). These results were consistent with the results of the lung function mentioned above and $125 \mathrm{mg} / \mathrm{kg}$ CAG was suggested to be an effective therapy for asthma.

It has been reported that CAG can regulate the levels of autophagy in myocardial cells (59), thus the present study investigated the probable binding between CAG and autophagy-associated targets, based on molecular docking. The results suggest that CAG may bind to the ATG4-LC3 complex to exert anti-inflammatory effects. Autophagy is the degradation of organelles and protein aggregates that are not degradable by proteasomes or invading microorganisms, such as viruses and bacteria (60). Autophagy-associated pathways and proteins play crucial roles in immunity and inflammation, acting as a central pivot to balance the beneficial and harmful effects of the host on infection and stimuli (61). Currently, the evaluation of autophagy is based on the autophagy markers, LC3, p62 and Beclin 1, which participate in the formation of autophagosome and phagophore (62). Previous studies have proposed that autophagy is promoted in the pathogenesis of asthma $(63,64)$, which is consistent with the results of the present study. The present study further investigated the modulation of autophagy by CAG in lung tissues of asthmatic mice. The results demonstrated that both 62.5 and $125 \mathrm{mg} / \mathrm{kg} \mathrm{CAG}$ reverted the increased protein expression levels of $\mathrm{LC} 3 \mathrm{~B}$ and restored the decreased protein expression levels of p62 in asthma. Notably, $125 \mathrm{mg} / \mathrm{kg}$ CAG triggered the regulation of autophagic flux to suppress autophagy, which might be associated with the attenuation of the development of asthma.

Due to the limitations of the experimental design, the present study only simulated the probable bond with CAG and autophagy-related targets, but failed to confirm their direct association, which can be verified via knockdown experiments. In addition, the mechanism by which cells express autophagy-related proteins, and are modulated by CAG in the lungs, remain unclear. 
In conclusion, the results of the present study verified CAG as a potential therapeutic target for AHR, airway inflammation and mucus hypersecretion in asthma, and suggested that these functions may be associated with the regulation of autophagic flux, mainly including decreased LC3B protein expression and increased p62 protein expression. However, further studies are required to confirm whether CAG alleviates airway inflammation by modulating autophagy. The results of the present study demonstrated that CAG exerted anti-inflammatory effects and inhibited autophagy in OVA-induced asthmatic murine, which provides the basis for further research on the target of CAG in the treatment of asthma.

\section{Acknowledgements}

Not applicable.

\section{Funding}

The present study was supported by the National Natural Science Foundation of China (grant no. 81774074), Shanghai Science and Technology Commission (grant nos. 17401930300 and 18401971300) and the Expert Workstation for Jingcheng Dong in Yunnan Province (grant no. 20210101).

\section{Availability of data and materials}

The datasets used and/or analyzed during the current study are available from the corresponding author on reasonable request.

\section{Authors' contributions}

$\mathrm{XZ}, \mathrm{YC}$ and JD designed the present study. XZ, MS, MC, CL, LY, JQ, WT, FT, YZ, WT and SW performed the experiments and confirmed the authenticity of all the raw data. XZ and YC analyzed the data and drafted the initial manuscript. JD revised the manuscript for important intellectual content. All authors have read and approved the final manuscript.

\section{Ethics approval and consent to participate}

All experimental procedures were approved by the Animal Care and Use Committee of the Fudan University (Shanghai, China; authorization no. 2018-10-HSYY-DJC-01).

\section{Patient consent for publication}

Not applicable.

\section{Competing interests}

The authors declare that they have no competing interests.

\section{References}

1. Cevhertas L, Ogulur I, Maurer DJ, Burla D, Ding M, Jansen K, Koch J, Liu C, Ma S, Mitamura Y, et al: Advances and recent developments in asthma in 2020. Allergy 75: 3124-3146, 2020.

2. Papi A, Brightling C, Pedersen SE and Reddel HK: Asthma. Lancet 391: 783-800, 2018.
3. Bao W, Zhang Y, Zhang M, Bao A, Fei X, Zhang X and Zhou X: Effects of ozone repeated short exposures on the airway/lung inflammation, airway hyperresponsiveness and mucus production in a mouse model of ovalbumin-induced asthma. Biomed Pharmacother 101: 293-303, 2018.

4. Busse W, Kraft M, Rabe KF, Deniz Y, Rowe PJ, Ruddy M and Castro M: Understanding the key issues in the treatment of uncontrolled persistent asthma with type 2 inflammation. Eur Respir J 58: 2003393, 2021

5. Piñeros YS, Bal SM, Dijkhuis A, Majoor CJ, Dierdorp BS, Dekker T, Hoefsmit EP, Bonta PI, Picavet D, van der Wel NN, et al: Eosinophils capture viruses, a capacity that is defective in asthma. Allergy 74: 1898-1909, 2019.

6. Reddel HK, Bateman ED, Becker A, Boulet LP, Cruz AA, Drazen JM, Haahtela T, Hurd SS, Inoue H, de Jongste JC, et al: A summary of the new GINA strategy: A roadmap to asthma control. Eur Respir J 46: 622-639, 2015.

7. Heffler E, Madeira LNG, Ferrando M, Puggioni F, Racca F, Malvezzi L, Passalacqua G and Canonica GW: Inhaled corticosteroids safety and adverse effects in patients with asthma. J Allergy Clin Immunol Pract 6: 776-781, 2018.

8. Xu Y and Eissa NT: Autophagy in innate and adaptive immunity. Proc Am Thorac Soc 7: 22-28, 2010.

9. Levine B, Mizushima N and Virgin HW: Autophagy in immunity and inflammation. Nature 469: 323-335, 2011.

10. Wang S, Wuniqiemu T, Tang W, Teng F, Bian Q, Yi L, Qin J, Zhu X, Wei Y and Dong J: Luteolin inhibits autophagy in allergic asthma by activating PI3K/Akt/mTOR signaling and inhibiting Beclin-1-PI3KC3 complex. Int Immunopharmacol 94: 107460, 2021.

11. Hill S, Wrobel L and Rubinsztein D: Post-translational modifications of Beclin 1 provide multiple strategies for autophagy regulation. Cell Death Differ 26: 617-629, 2019.

12. Matsuzawa-Ishimoto Y, Hwang S and Cadwell K: Autophagy and Inflammation. Ann Rev Immunol 36: 73-101, 2018.

13. Kirkin V, McEwan D, Novak I and Dikic I: A role for ubiquitin in selective autophagy. Mol Cell 34: 259-269, 2009.

14. Deretic V: Autophagy in inflammation, infection, and immunometabolism. Immunity 54: 437-453, 2021

15. Racanelli AC, Kikkers SA, Choi AMK and Cloonan SM: Autophagy and inflammation in chronic respiratory disease. Autophagy 14: 221-232, 2018.

16. Renz H: Autophagy: Nobel prize 2016 and allergy and asthma research. J Allergy Clin Immunol 140: 1548-1549, 2017.

17. Silveira JS, Antunes GL, Kaiber DB, da Costa MS, Ferreira FS, Marques EP, Schmitz F, Gassen RB, Breda RV, Wyse ATS, et al: Autophagy induces eosinophil extracellular traps formation and allergic airway inflammation in a murine asthma model. J Cell Physiol 235: 267-280, 2020.

18. Liu JN, Suh DH, Trinh HK, Chwae YJ, Park HS and Shin YS: The role of autophagy in allergic inflammation: A new target for severe asthma. Exp Mol Med 48: e243, 2016.

19. McAlinden KD, Deshpande DA, Ghavami S, Xenaki D, Sohal SS, Oliver BG, Haghi M and Sharma P: Autophagy activation in asthma airways remodeling. Am J Respir Cell Mol Biol 60: $541-553,2019$.

20. Chan HHL and $\mathrm{Ng}$ T: Traditional Chinese medicine (TCM) and allergic diseases. Curr Allergy Asthma Rep 20: 67, 2020.

21. Wang W, Liu QB and Jing W: Astragalus membranaceus improves therapeutic efficacy of asthmatic children by regulating the balance of Treg/Th17 cells. Chin J Nat Med 17: 252-263, 2019.

22. Wang W, Jing W and Liu Q: Astragalus oral solution ameliorates allergic asthma in children by regulating relative contents of $\mathrm{CD} 4^{+} \mathrm{CD} 25^{\text {high }} \mathrm{CD} 127^{\text {low }}$ treg cells. Front Pediatr 6: 255, 2018.

23. Li L, Hou X, Xu R, Liu C and Tu M: Research review on the pharmacological effects of astragaloside IV. Fundam Clin Pharmacol 31: 17-36, 2017.

24. Li K, Chen Y, Jiang R, Chen D, Wang H, Xiong W, Li D, Liu Z, Li X, Li J and Yuan K: Protective effects of astragaloside IV against ovalbumin-induced allergic rhinitis are mediated by T-box protein expressed in T cells/GATA-3 and forkhead box protein 3/retinoic acid-related orphan nuclear receptor $\gamma \mathrm{t}$. Mol Med Rep 16: 1207-1215, 2017.

25. Huang X, Tang L, Wang F and Song G: Astragaloside IV attenuates allergic inflammation by regulation $\mathrm{Th} 1 / \mathrm{Th} 2$ cytokine and enhancement CD4(+)CD25(+)Foxp3 T cells in ovalbumin-induced asthma. Immunobiology 219: 565-571, 2014.

26. Calis I, Gazar H, Piacente S and Pizza C: Secondary metabolites from the roots of Astragalus zahlbruckneri. J Nat Prod 64: 1179-1182, 2001. 
27. Yu Y, Zhou L, Yang Y and Liu Y: Cycloastragenol: An exciting novel candidate for age-associated diseases. Exp Ther Med 16: 2175-2182, 2018.

28. Wan Y, Xu L, Wang Y, Tuerdi N, Ye M and Qi R: Preventive effects of astragaloside IV and its active sapogenin cycloastragenol on cardiac fibrosis of mice by inhibiting the NLRP3 inflammasome. Eur J Pharmacol 833: 545-554, 2018.

29. Melgert BN, Postma DS, Kuipers I, Geerlings M, Luinge MA, van der Strate BW, Kerstjens HAM, Timens W and Hylkema MN: Female mice are more susceptible to the development of allergic airway inflammation than male mice. Clin Exp Allergy 35: 1496-1503, 2005.

30. Li Y, Chen S, Chi Y, Yang Y, Chen X, Wang H, Lv Z, Wang J, Yuan L, Huang P, et al: Kinetics of the accumulation of group 2 innate lymphoid cells in IL-33-induced and IL-25-induced murine models of asthma: A potential role for the chemokine CXCL16. Cell Mol Immunol 16: 75-86, 2019.

31. Peng H, Ning H, Wang Q, Lu W, Chang Y, Wang TT, Lai J, Kolattukudy PE, Hou R, Hoft DF, et al: Monocyte chemotactic protein-induced protein 1 controls allergic airway inflammation by suppressing IL-5-producing $\mathrm{T}_{\mathrm{H}} 2$ cells through the Notch/Gata3 pathway. J Allergy Clin Immunol 142: 582-594, 2018.

32. Chen S, Yun F, Yao Y, Cao M, Zhang Y, Wang J, Song X and Qian Y: USP38 critically promotes asthmatic pathogenesis by stabilizing JunB protein. J Exp Med 215: 2850-2867, 2018.

33. Tel BC, Telli G, Onder S, Nemutlu E and Bozkurt TE: Investigation of the relationship between chronic montelukast treatment, asthma and depression-like behavior in mice. Exp Ther Med 21: 27, 2021.

34. Tamaru S, Mishina H, Watanabe Y, Watanabe K, Fujioka D, Takahashi S, Suzuki K, Nakamura T, Obata JE, Kawabata K, et al: Deficiency of phospholipase A2 receptor exacerbates ovalbumin-induced lung inflammation. J Immunol 191: 1021-1028, 2013

35. Kim S, Thiessen PA, Bolton EE, Chen J, Fu G, Gindulyte A, Han L, He J, He S, Shoemaker BA, et al: PubChem substance and compound databases. Nucleic Acids Res 44: D1202-D1213, 2016.

36. Berman H, Westbrook J, Feng Z, Gilliland G, Bhat TN, Weissig H, Shindyalov IN and Bourne PE: The protein data bank. Nucleic Acids Res 28: 235-242, 2000.

37. Morris G, Huey R, Lindstrom W, Sanner MF, Belew RK, Goodsell DS and Olson AJ: AutoDock4 and AutoDockTools4: Automated docking with selective receptor flexibility. J Comput Chem 30: 2785-2791, 2009.

38. Trott $\mathrm{O}$ and Olson A: AutoDock vina: Improving the speed and accuracy of docking with a new scoring function, efficient optimization, and multithreading. J Comput Chem 31: 455-461, 2010.

39. Seeliger D and de Groot B. Ligand docking and binding site analysis with PyMOL and autodock/vina. J Comput Aided Mol Des 24: 417-422, 2010.

40. Wang C, Tu M, Wu D, Chen $\mathrm{H}$, Chen $\mathrm{C}$, Wang $\mathrm{Z}$ and Jiang L: Identification of an ACE-inhibitory peptide from walnut protein and its evaluation of the inhibitory mechanism. Int J Mol Sci 19 1156, 2018.

41. Feng FB and Qiu HY: Effects of Artesunate on chondrocyte proliferation, apoptosis and autophagy through the PI3K/AKT/mTOR signaling pathway in rat models with rheumatoid arthritis. Biomed Pharmacother 102: 1209-1220, 2018.

42. Wei Y, Liu B, Sun J, Lv Y, Luo Q, Liu F and Dong J: Regulation of Th17/Treg function contributes to the attenuation of chronic airway inflammation by icariin in ovalbumin-induced murine asthma model. Immunobiology 220: 789-797, 2015.

43. Tang W, Dong M, Teng F, Cui J, Zhu X, Wang W, Wuniqiemu T, Qin J, Yi L, Wang S, et al: TMT-based quantitative proteomics reveals suppression of SLC3A2 and ATP1A3 expression contributes to the inhibitory role of acupuncture on airway inflammation in an OVA-induced mouse asthma model. Biomed Pharmacother 134: 111001, 2021.

44. Kubo M: Innate and adaptive type 2 immunity in lung allergic inflammation. Immunol Rev 278: 162-172, 2017.

45. Zhu X, Cui J, Yi L, Qin J, Tulake W, Teng F, Tang W, Wei Y and Dong $\mathrm{J}$ : The role of $\mathrm{T}$ cells and macrophages in asthma pathogenesis: A new perspective on mutual crosstalk. Mediators Inflamm 2020: 7835284, 2020.
46. Bleecker ER, Menzies-Gow AN, Price DB, Bourdin A, Sweet S, Martin AL, Alacqua M and Tran TN: Systematic literature review of systemic corticosteroid use for asthma management. Am J Respir Crit Care Med 201: 276-293, 2020.

47. Zhu J, Lee S, Ho MK, Hu Y, Pang H, Ip FC, Chin AC, Harley CB, Ip NY and Wong YH: In vitro intestinal absorption and first-pass intestinal and hepatic metabolism of cycloastragenol, a potent small molecule telomerase activator. Drug Metab Pharmacokinet 25: 477-486, 2010.

48. Li M, Li SC, Dou BK, Zou YX, Han HZ, Liu DX, Ke ZJ and Wang ZF: Cycloastragenol upregulates SIRT1 expression, attenuates apoptosis and suppresses neuroinflammation after brain ischemia. Acta Pharmacol Sin 41: 1025-1032, 2020.

49. Gu M, Zhang S, Zhao Y, Huang J, Wang Y, Li Y, Fan S, Yang L, Ji G, Tong Q and Huang C: Cycloastragenol improves hepatic steatosis by activating farnesoid $\mathrm{X}$ receptor signalling. Pharmacol Res 121: 22-32, 2017.

50. Wang Y, Chen C, Wang Q, Cao Y, Xu L and Qi R: Inhibitory effects of cycloastragenol on abdominal aortic aneurysm and its related mechanisms. Br J Pharmacol 176: 282-296, 2019.

51. Liu J, Gao D, Dan J, Liu D, Peng L, Zhou R and Luo Y: The protective effect of cycloastragenol on aging mouse circadian rhythmic disorder induced by d-galactose. J Cell Biochem 120: 16408-16415, 2019.

52. Deng G, Chen W, Wang P, Zhan T, Zheng W, Gu Z, Wang X, Ji X and Sun Y: Inhibition of NLRP3 inflammasome-mediated pyroptosis in macrophage by cycloastragenol contributes to amelioration of imiquimod-induced psoriasis-like skin inflammation in mice. Int Immunopharmacol 74: 105682, 2019.

53. Kanehiro A, Takeda K, Joetham A, Tomkinson A, Ikemura T, Irvin CG and Gelfand EW: Timing of administration of anti-VLA-4 differentiates airway hyperresponsiveness in the central and peripheral airways in mice. Am J Respir Crit Care Med 162: 1132-1139, 2000.

54. Dong L, Wang Y, Zheng T, Pu Y, Ma Y, Qi X, Zhang W, Xue F, Shan Z, Liu J, et al: Hypoxic hUCMSC-derived extracellular vesicles attenuate allergic airway inflammation and airway remodeling in chronic asthma mice. Stem Cell Res Ther 12: 4 , 2021.

55. Hammad $\mathrm{H}$ and Lambrecht BN: The basic immunology of asthma. Cell 184: 1469-1485, 2021.

56. Chen W, Sivaprasad U, Gibson AM, Ericksen MB, Cunningham CM, Bass SA, Kinker KG, Finkelman FD, Wills-Karp M and Hershey GK: IL-13 receptor $\alpha 2$ contributes to development of experimental allergic asthma. J Allergy Clin Immunol 132: 951-958, 2013.

57. Lambrecht BN, Hammad H and Fahy JV: The cytokines of asthma. Immunity 50: 975-991, 2019.

58. Gould HJ and Sutton BJ: IgE in allergy and asthma today. Nat Rev Immunol 8: 205-217, 2008.

59. Wang J, Wu ML, Cao SP, Cai H, Zhao ZM and Song YH: Cycloastragenol ameliorates experimental heart damage in rats by promoting myocardial autophagy via inhibition of AKT1-RPS6KB1 signaling. Biomed Pharmacother 107: 1074-1081, 2018.

60. Cadwell K: Crosstalk between autophagy and inflammatory signalling pathways: Balancing defence and homeostasis. Nat Rev Immunol 16: 661-675, 2016.

61. Mizushima N and Levine B: Autophagy in human diseases. N Engl J Med 383: 1564-1576, 2020.

62. Maneechotesuwan K, Kasetsinsombat K, Wongkajornsilp A and Barnes PJ: Role of autophagy in regulating interleukin-10 and the responses to corticosteroids and statins in asthma. Clin Exp Allergy 19: 13825, 2021.

63. Xia F, Deng C, Jiang Y, Qu Y, Deng J, Cai Z, Ding Y, Guo Z and Wang J: IL4 (interleukin 4) induces autophagy in B cells leading to exacerbated asthma. Autophagy 14: 450-464, 2018.

64. Zhang Y, Do DC, Hu X, Wang J, Zhao Y, Mishra S, Zhang X, Wan $M$ and Gao P: CaMKII oxidation regulates cockroach allergen-induced mitophagy in asthma. J Allergy Clin Immunol 147: 1464-1477, 2021

This work is licensed under a Creative Commons Attribution-NonCommercial-NoDerivatives 4.0 International (CC BY-NC-ND 4.0) License. 